\title{
Development of a Robotic System to Enable Beating- heart Surgery
}

\author{
Meaghan Bowthorpe*1, Vincent Castonguay-Siu*2 and Mahdi Tavakoli*1 \\ ${ }^{* 1}$ Department of Electrical and Computer Engineering, University of Alberta \\ ${ }^{*}$ Department of Mechanical Engineering, University of Alberta
}

\section{Introduction}

Cardiovascular disease causes the greatest number of deaths worldwide each year according to the World Health Organization [1]. Hence, many patients will require cardiovascular surgery each year. Short of stopping the motion of the heart at the beginning of an extra-cardiac procedure, the surgeon would require superhuman ability to both follow the heart's beating motion and perform surgical maneuvers on the exterior surface of the heart. Performing a surgical procedure within the interior of a beating heart would be even more difficult due to the opaque blood pool. Currently, to overcome the aforementioned obstacles, surgeons operate on either a mechanically stabilized or arrested heart, where a heart-lung machine ventilates the lungs and circulates the blood. A mechanical heart stabilizer can only minimize motion in a localized area on the exterior surface of the heart; it cannot completely stop the motion. Arresting the heart can lead to long-term cognitive loss [2], an increase in the risk of stroke [3], and complications when the heart is restarted.

Alternatively, a robot-held surgical tool can be controlled to follow the motion of a point of interest (POI) on the heart, allowing the heart to beat freely throughout the procedure. In this scenario, the surgeon's hand motion is superimposed on this synchronization movement. In this way, with a stabilized view of the heart, the heart appears stationary to the surgeon. Allowing

原稿受付 2014 年 3 月 17 日

キーワード : Beating-heart Surgery, Robotic-assistance, Ultrasound Image Guidance

*1,2 Edmonton, Alberta, Canada T6G 2V4

This work was supported by the Natural Sciences and Engineering Research Council (NSERC) of Canada, the Canada Foundation for Innovation (CFI), a Dean's Research Award from the Faculty of Engineering at the University of Alberta, and a Queen Elizabeth II Graduate Student Scholarship from the Government of Alberta. the heart to beat freely during the procedure means that the surgeon will be able to immediately evaluate the success of reconstructive operations and make adjustments as required. In contrast, using the current practice of arresting the heart at the outset of surgery, the outcome of the procedure is not known until after restarting, and the surgeon would have to once again connect the patient to a heart-lung machine should additional operations be required.

\section{Prior Art}

To develop a beating-heart surgical system where the surgical tool follows the combined motion of the surgeon and the heart, two software-based elements are necessary. The first element keeps track of the positions of the surgical tool and the beating heart. This can be done by continually taking images of the heart and surgical tool with a camera or medical scanner. High-speed cameras can be used to visualize the exterior surface of the heart [4] and ultrasound images can be used to visualize both the exterior and the interior surfaces of the heart [5]. In addition, sonomicrometry crystals can be sutured to the heart to track the position of a specific point on the heart [6]; as it is not practical to suture sonomicrometry crystals to the heart during surgery, this method is more appropriate for validation of control techniques.

The second element controls the surgical tool to follow the motion of the beating heart. Choosing the type of controller depends on whether the first element's measurement of the surgical tool and beating heart positions introduces a delay. When a high-speed camera captures the positions, a generalized predictive controller can be used [4]. If a delay is introduced by the first element, however, the current position of the heart must be estimated. This can be accomplished with an extended Kalman filter [7] or by temporally adjusting the previous 
heart beat motion to match the current heart rate [6] Depending on whether the surgical robot is directly held by the surgeon or is teleoperated from a user interface, there may be a need for additional delay compensation steps.

This research focuses on procedures performed on both the interior and the exterior surfaces of the heart. As camera images cannot visualize through the opaque blood pool, they cannot be used. Instead, ultrasound images are used in the first software-based element to calculate the heart's and the surgical tool's motions. As it takes time to obtain and process each ultrasound image frame, a non-negligible time delay is introduced. This has detrimental effects on the system stability and performance, and must therefore be compensated for by the second software-based element - the control system. This control system is designed to ensure that the surgical tool follows the combination of the heart's motion and the surgeon's motion in the presence of delayed measurements of the heart and the surgical tool positions. Since the surgical tool is teleoperated (as opposed to being directly held by the surgeon), a Smith predictor is used for delay compensation as part of the control system.

The remainder of this paper is organized as follows. The control framework of the surgical system including motivating surgical procedures, the structure of the image-based motion tracking, and the control system is discussed in Sec. III. The design of the experimental testbed is given in Sec. IV including the structure of the surgical tool and the design of a mechanical simulator for a beating heart. Sec. V presents the experimental results concerning the system performance and stability. Concluding remarks are given in Sec. VI.

\section{System Description}

To successfully design a robot-assisted beating heart surgical system, several requirements must be met. To facilitate procedures on both the interior and exterior surfaces of the heart, the method of visualizing the surgical tool and the heart must be able to "see" through the opaque blood pool. For this reason, ultrasound images will be used. However, this introduces a nonnegligible time delay of approximately 40 [ms] into the system for image acquisition. This delay is then lengthened due to the time needed for processing each image where the location of the surgical tool tip and the POI on the heart is calculated. Because of this, the control system responsible for the motion of the surgical tool must be able to compensate for the total delay.

\section{A. Motivating Surgical Procedures}

Many surgical procedures can benefit from a robotassisted beating-heart surgical system. Even surgical procedures performed on organs other than the heart that are subject to periodic respiratory motions can benefit. For example, radiation treatments can be delivered more precisely if the laser beam moves in synchrony with the motion of the affected organ [8]. This synchronization is more difficult for procedures performed on the heart as the heart's motion is composed of fast and small heartbeat-induced motion as well as the slow and large respiration-induced motion.

This research focuses solely on the more difficult case of compensating for the heartbeat-induced motion. Initially, surgical procedures requiring one-dimensional motion tracking are considered. These include pericardiocentesis, where a needle is inserted into the pericardial sac surrounding the heart to drain the excess fluid. During this procedure, the needle should remain within the pericardial sac but should not puncture any blood vessels on the surface of the heart. Motion synchronization can aid in preventing this type of a hazard. Mitral valve annuloplasty also requires one-dimensional motion tracking [9]. The surgeon can reshape the mitral valve annulus by stapling an annuloplasty ring within it and evaluate the performance of the newly shaped valve on the fly.

\section{B. Ultrasound Image-Based Tissue Tracking}

Ultrasound imaging is used to locate the POI on the heart and the surgical tool and was chosen because it can visualize the interior of the heart through the opaque blood pool and is less invasive when visualizing the exterior surface of the heart. During the surgical procedures, ultrasound images are continually gathered at a fixed rate. Each image frame is then processed to determine the location of the surgical tool and the heart as is shown in Fig. 1. The ultrasound image acquisition and processing adds a time delay of approximately 150 [ms] [7] into the system. This delay must be later compensated for by the controller; otherwise, the surgical tool may collide with the heart.

Once an image is collected, a Radon transform [5] is used to find the location of the longest straight line in the image, which corresponds to the surgical tool. Next, 


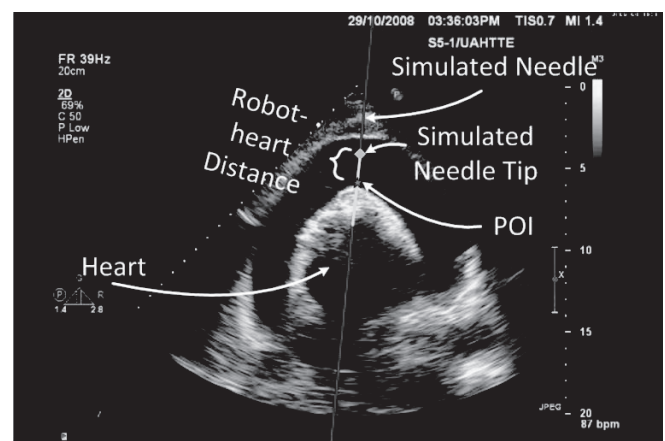

Fig. 1 An ultrasound image of the heart and a simulated surgical tool (needle). First, the axis of the surgical tool is found and extended across the entire image. Then, moving along this axis the location of the surgical tool tip and the closest heart tissue is found.

the axis of this line is extended across the image. The POI is simply the closest heart tissue directly in front of the surgical tool. The location of the POI is found by calculating the derivative of the pixel values along the surgical tool axis; the peak value of this derivative marks a change from a dark region (fluid-filled) to a bright region (tissue). Now that the location of the surgical tool and POI are both known, the distance between them can be calculated. The goal is to control this distance to follow the surgeon's motion.

\section{Feedback Control of Surgical Tool}

The feedback control system is responsible for synchronizing the movement of the surgical tool with that of the POI, and additionally superimposing the surgeon's motion onto the surgical tool's motion. In other words, the feedback controller should be designed where the input (setpoint) is the surgeon's position and the output (controlled variable) is the distance between the POI and the surgical tool. As mentioned before, a complicating factor is that measuring the positions of the POI and the surgical tool is subject to delays due to the ultrasound image acquisition and processing. Using the delayed measurement in the feedback loop may cause instability and lead to collisions between the heart and the surgical tool.

In this work, a Smith predictor is used to compensate for this delay by effectively removing it from within the feedback loop [10]. The assumptions are that the surgical robot (plant) dynamical model and the delay value are known or estimated. Consider the feedback loop with setpoint $R$, controller $\bar{C}$, plant $G$, delay $e^{-s L}$, and output $Y$ in Fig. 2 (a). For delay compensation in this

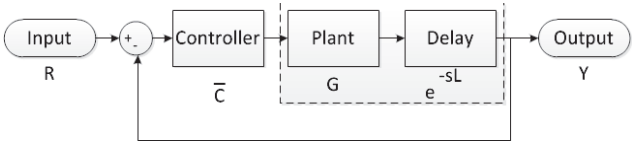

(a)

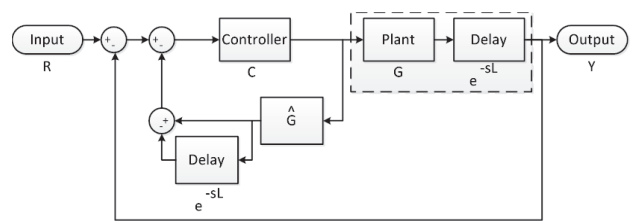

(b)

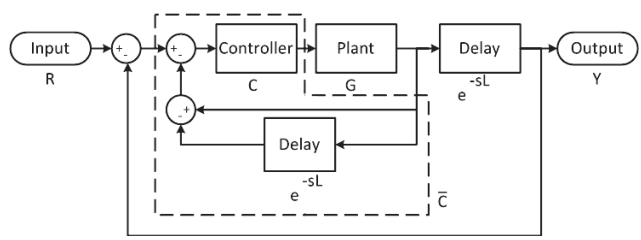

(c)

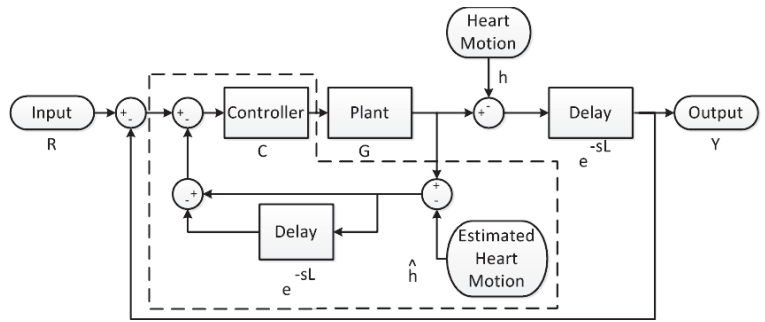

(d)

Fig. 2 Diagrams (a) through (d) show the development of a Smith predictor controller. (a) A feedback loop with a time delay. (b) The same feedback loop with the addition of a Smith predictor controller. (c) The Smith predictor is altered because the actual output of plant $G$ is accessible. (d) The final Smith predictor controller where the actual and estimated heart motion has been included.

feedback loop, an estimated model of $G$, called $\hat{G}$, is used in an internal feedback loop in Fig. 2 (b). To cancel the effect of the external delayed feedback loop, this internal loop also includes the delayed version of the estimated plant's output as shown in Fig. 2 (b). If $G$ and $\hat{G}$ are the same and the delay is known exactly, then the effect of the external loop is cancelled and it will be as if the plant output is fed back without experiencing any delay.

The feedback loop with controller $C$, plant $G$, output $Y$, and setpoint $R$ can be described by the following closed-loop transfer function $H$ :

$$
H=\frac{Y}{R}=\frac{C G}{1+C G}
$$

Assume that, in the time-delayed case, we are using a controller in which case the closed-loop transfer function, $\bar{H}$, will be 


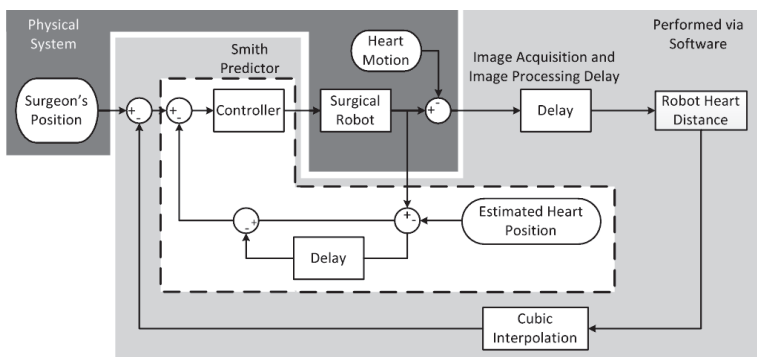

Fig. 3 The final model of the Smith predictor based control loop to ensure that the distance between the surgical tool and the POI follows the surgeon's motion.

$$
\bar{H}=\frac{Y}{R}=\frac{\bar{C} G}{1+\bar{C} G},
$$

The key observation in the Smith predictor design is that in order to retain the performance of the nondelayed system, $\bar{H}$ must be equal to $H$ multiplied by the time delay. This means that $\bar{C}$ is simply calculated by multiplying Exp. (1) by the Laplace transform of the time delay and then equating to Exp. (2). Solving for $\bar{C}$ we obtain

$$
\bar{C}=\frac{C}{1+C G\left(1-e^{-s L}\right)} .
$$

Note that the above expression for $\bar{C}$ matches the Smith predictor's control system shown in Fig. 2 (b).

This research is a special case of this scenario where the delay is caused by the imaging. While the distance between the positions of the POI and the surgical tool is only known after the delay, the surgical robot's position is known in real-time (from robot encoders or external trackers). Therefore, we employ a modification of the Smith predictor shown in Fig. 2 (c) that has the same functionality as the system in Fig. 2(b). A further necessary modification is shown in Fig. 3: The "current" position of the POI (Heart Motion block), only the delayed value of which we measure, acts as a disturbance in the feedback loop in Fig. 3. Hence, a "prediction" of the current position of the POI (Estimated Heart Motion block) is added to the interior feedback loop of the Smith predictor. In this way, the Smith predictor compensates for the time delay in acquiring the distance between the POI and the surgical tool by using the real-time position of the surgical tool and estimating the POI's current position (e.g., based on past POI position and electrocardiography signals). For more details about the Smith predictor, see Ref. [11].

Next, the heart motion is modeled as a disturbance $h$.
The sensitivity function of such a disturbance if introduced after the plant in the non-delayed feedback loop shown in Fig. 2 (a) would be

$$
Y=\frac{-h}{1+C G}
$$

To maintain this same sensitivity function once the delay is included in the system, an estimate of the current heart motion is added to the inner feedback loop as is shown in Fig. 2 (d). If the estimated heart motion $\hat{h}$ is the same as the actual heart motion $h$, the sensitivity function will reduce to the following

$$
Y=\frac{-h e^{-s L}}{1+C G}+\frac{(\hat{h}-h)\left(1-e^{-s L}\right) C G e^{-s L}}{1+C G}=\frac{-h e^{-s L}}{1+C G}
$$

which is the equivalent to Exp. (4) multiplied by the delay. The complete model of the feedback control system is shown in Fig. 3. In the following, we discuss a method for obtaining estimates of the current position of the POI.

As the current position of the heart is not known in real-time, it must be estimated for use in Fig. 2 (d). The estimation method chosen takes advantage of the periodicity of the heart's beating motion. The current position of the POI is estimated to be the position of the POI in the previous heart beat.

\section{Experimental Testbed}

The experimental testbed includes the (motorized) surgical tool shown in Fig. 5, a Micron Tracker (model HX60 from Claron Technology Inc., Toronto, Ontario, Canada), and a mechanical heart simulator shown in Fig. 9. The surgical tool as explained below allows for the synchronization between the surgical tool's tip and the POI on the beating heart along one dimension. The Micron Tracker is used to simulate an ultrasound scanner. Its frame rate of $20[\mathrm{~Hz}]$ is similar or lower than the frame rate of an ultrasound scanner. The Micron Tracker also has a lag of $85[\mathrm{~ms}]$ and an image processing delay of 20 [ms]. Therefore, the complete image acquisition and processing delay is approximately 105 [ms]. This is similar to the 150 [ms] image acquisition and processing delay of ultrasound images found by Yuen et al. [7]. A mechanical cam designed based on the movement of a single point on the exterior of the heart calculated from ultrasound images is used to represent the POI on the beating heart. 


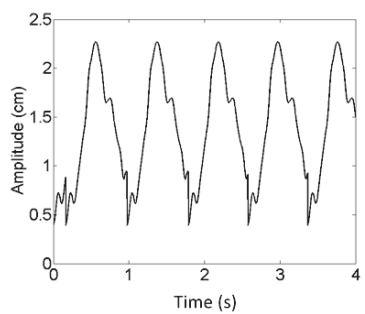

(a)

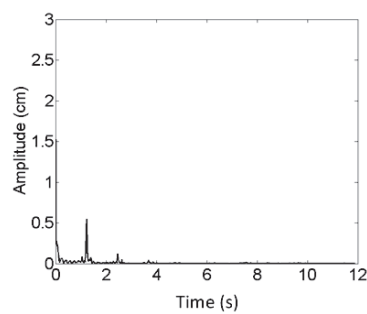

(b)
Fig. 4 (a) The motion of a heart beat taken from ultrasound images and temporally matched to an ECG signal to create a series of heart beats. (b) The frequency spectrum of the heart beats.

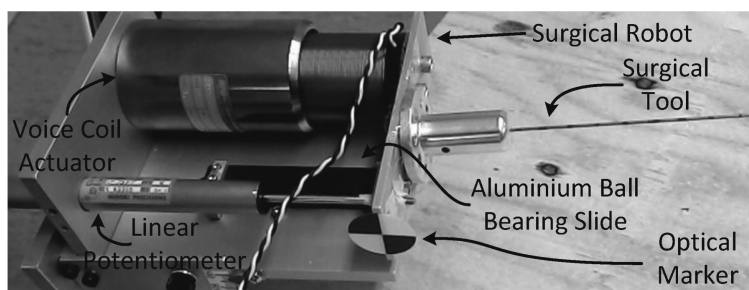

Fig. 5 A close up view of the surgical tool, which is moved by a voice coil actuator. The aluminium ballbearing linear slide confines the motion along a single dimension. A linear potentiometer measures the position of the voice coil actuator in real-time. The optical marker is required for the Claron Microtracker to measure the position of the voice coil actuator.

\section{A. Ultrasound Image and Heart Motion Sam- pling Rate}

The choice of the sampling time used to acquire images and control the surgical robot merits further discussion. The image acquisition sampling time is limited to $50[\mathrm{~ms}]$ or $20[\mathrm{~Hz}]$ due to the frame rate of the Micron Tracker. To determine the sampling speed required to control the surgical tool, a spectral decomposition of the heart's beating motion is required. Fig. 4 (a) shows the shape of the motion of a POI on beating heart taken from ultrasound images and the frequencies present in the motion are given in Fig. 4(b). The highest frequency component is less than $4[\mathrm{~Hz}]$. To follow this motion the minimum sampling frequency according to the Nyquist theorem is $8[\mathrm{~Hz}]$ however, it is better to have a much higher sampling rate. Yuen et al. reported the highest frequency component in the motion of the mitral valve to be approximately $12[\mathrm{~Hz}][12]$ and would require a minimum sampling frequency of 24 [Hz]. Better tracking is possible if the sampling frequency is approximately eight times greater than the maximum frequency present, which is $96[\mathrm{~Hz}]$ for the case of the mitral valve's motion. For this research, a sampling frequency of $100[\mathrm{~Hz}]$ is chosen.

Because a sample rate of $20[\mathrm{~Hz}]$ is not fast enough, the slowly sampled signals, which include the measurement of the position of the POI and the position of the tool by the Micron Tracker, must be upsampled. Two methods are chosen: zero-order-hold and cubic interpolation. In the case of a zero-order-hold, the newest measurement value is kept until a new measurement is available. This method does not affect the delay in the system. In the case of cubic interpolation new values are inserted in between two consecutive measurements (end points) in such a way that the derivative of the measurements at each end point and the value of each end point are preserved. The following equations describe this process.

$$
p(t)=h_{00}(t) p_{0}+h_{10}(t) m+h_{01}(t) p_{1}+h_{11}(t) m_{1}
$$

$$
\begin{gathered}
h_{00}(t)=2 t^{3}-3 t^{2}+1 \\
h_{10}(t)=t^{3}-3 t^{2}+t \\
h_{01}(t)=-2 t^{3}+t^{2} \\
h_{11}(t)=t^{3}-t^{2}
\end{gathered}
$$

where $p(t)$ is the interpolated point, $p 0$ and $p 1$ are the two end points between which the interpolation is occurring, $m 0$ and $m 1$ are the slopes at the two end points, and $t$ is a value between 0 and 1 . As four sample points are required to measure the slopes at each end point, this method increases the delay by the length of two slowly sampled signals.

\section{B. Surgical Robot Structure}

The surgical robot is designed for synchronizing the motion of a surgical tool (needle) with the heart's motion along a single linear dimension. The surgical tool must have a range of a few centimeters and have the ability to move with velocities of $210[\mathrm{~mm} / \mathrm{s}]$ and accelerations of $3,800\left[\mathrm{~mm} / \mathrm{s}^{2}\right][9]$ in order to keep up with the peak velocities and accelerations of the heart.

To meet these requirements, a voice coil actuator (NCC20-18-020-1X from H2W Technologies Inc., Santa Clarita, CA, USA) was chosen to create the necessary back-and-forth motions of the surgical tool. An aluminum ball-bearing linear slide (6203K89 from McMaster-Carr, Aurora, OH, USA) is used to ensure this motion stays along one dimension. To measure the displacement of the surgical tool (and the voice coil actuator) in real-time, a linear potentiometer (LP-75FP 


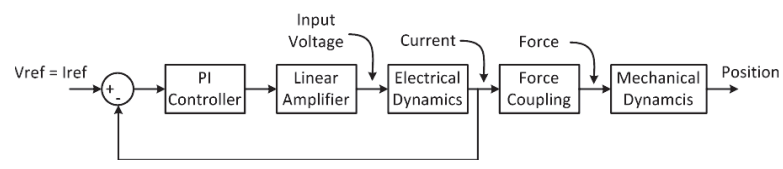

Fig. 6 The electrical and mechanical characteristics of the analog controller that controls the position of the voice coil actuator.

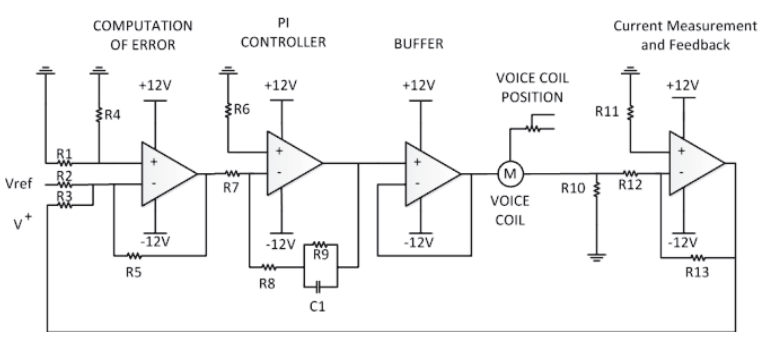

Fig. 7 The circuit diagram of the analog control loop responsible for the current control of the voice coil actuator.

from Midori America Corp., Fullerton, CA, USA) is mounted on the robot.

The motion of the voice coil is controlled by applying a voltage across the coil. The resulting current creates a magnetic field which either pushes the coil out of or into the cylindrical permanent magnet acting as the actuator's base. This motive force will be proportional to the current passing through the coil, thus necessitating an internal current control loop as part of any system for feedback control of the voice coil's position. A block diagram of the steps involved in controlling the position of the voice coil is given in Fig. 6 and the circuit diagram is shown in Fig. 7. This circuit has four stages: the computation of error, the proportional-integral (PI) controller, the buffer, and the current measurement and feedback. The first section of this circuit calculates the error between the reference voltage $V_{\text {ref }}$ representing the desired current and the voltage $V_{+}$that as explained later is proportional to the current passing through the voice coil. The second section generates PI action based on this error. If the output of the PI controller is connected directly to the voice coil, there will be a loading effect meaning that the voltage will be reduced once current is drawn. Therefore, the third stage of the circuit which is a buffer (a linear amplifier from Quanser Inc. Markham, Ontario, Canada) ensures that the voltage remains unchanged while supplying a sufficient current to the voice coil. Finally, the actual current passing through the coil is calculated by measuring the voltage across a small $(0.3[\Omega])$ resistor placed in series with the

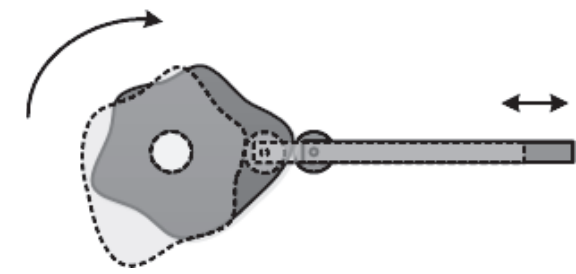

Fig. 8 This mechanical cam converts rotational motion into linear motion. As the cam rotates, it moves the rectangular arm linearly.

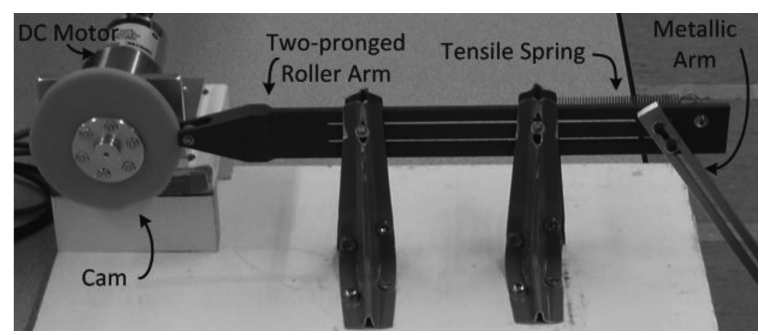

Fig. 9 The mechanical oscillator built to mimic the motion of a single point on the heart. A mechanical cam is used to convert rotational motion into linear motion.

coil and amplifying it using the fourth stage of the circuit to the range between $-10[\mathrm{~V}]$ to $10[\mathrm{~V}]$. The output is the measured voltage $V_{+}$used for the initial error calculation in the first stage of the circuit.

\section{Simulated Heart}

A mechanical oscillator was built to mimic the movements experienced by a single point on a human heart. The cyclic oscillation to be replicated was extracted from the actual motion of a single point on the exterior heart wall from a series of ultrasound images. The final design utilized a mechanical cam to convert rotational movement of a DC motor into linear movement which would then be extended into a large container full of water using arm links (a section of the device is to be used underwater whilst permitting clear visualization under ultrasound). The mechanical oscillators target displacement away from its start position was set to approximately $1[\mathrm{~cm}]$ while it must have the potential to reach a maximal displacement of $3[\mathrm{~cm}]$ with only minor adjustments to the cam. A diagram of a mechanical cam is shown in Fig. 8. As the cam rotates about its axis, the rectangular arm is moved back and forth in a way that is dependent on the cam's shape. The cylindrical cam is connected to a DC motor (DC Gearmotor 6331K33 from McMaster-Carr, Aurora, OH, USA) permitting control over the cam's speed and torque. The final mechanical design is shown in Fig. 9. 


\section{Experimental Results}

The experimental testbed is used to show the usefulness and necessity of the Smith predictor. The position of the surgical tool and the heart are measured by the Micron Tracker at a rate of $20[\mathrm{~Hz}]$. However, to follow the motion of the heart, the surgical tool must be controlled at a higher rate. Both the real-time position measurements of the tool by the potentiometer and the control of the surgical tool occur at a rate of $100[\mathrm{~Hz}]$. The delayed and estimated heart positions as well as the tool position measurements are upsampled using either a zero-order-hold or cubic interpolation; however, the distance between the tool and the POI (the system's output) is not upsampled. Initially, the Smith Predictor is removed from the system and only the controller $C$ is used in the delayed feedback loop. The results are given in Fig. 10, where the solid line is the distance between the surgical tool and the heart and the dashed line is the surgeon's motion. The tool moved to its limit point and remained there. This performance is not acceptable. Next, the Smith predictor is returned to the system and the slow data signals are upsampled using a zero-order-hold. The actual and predicted heart motion are shown in Fig. 11 by the dashed and solid lines respectively. The distance between the tool and the heart and the surgeon's motion are shown in Fig. 12 by the solid and dashed lines respectively. In this case the distance between the tool and the heart follows the surgeon's motion with a delay. The mean absolute error and the integrated squared error (ISE), which is $\Sigma \epsilon^{2} / n$ where $\varepsilon$ is the error value and $\mathrm{n}$ is the number of data points, between the surgeon's motion and the distance between the tool and the POI are $2.9[\mathrm{~mm}]$ and $6.0\left[\mathrm{~mm}^{2}\right]$ respectively. Next, cubic interpolation is used to upsample the slow data signals. The actual and predicted heart motion are shown in Fig. 13 by the dashed and solid lines respectively. The distance between the tool and the heart and the surgeon's motion are shown in Fig. 14 by the solid and dashed lines respectively. The mean absolute error and ISE between the surgeon's motion and the distance between the tool and the POI are $0.55[\mathrm{~mm}]$ and $0.80\left[\mathrm{~mm}^{2}\right]$ respectively, a noticeable improvement over the previous case. In this case the distance between the tool and the heart follows the surgeon's motion more closely than the zero-orderhold case except that the delay is longer. In both cases

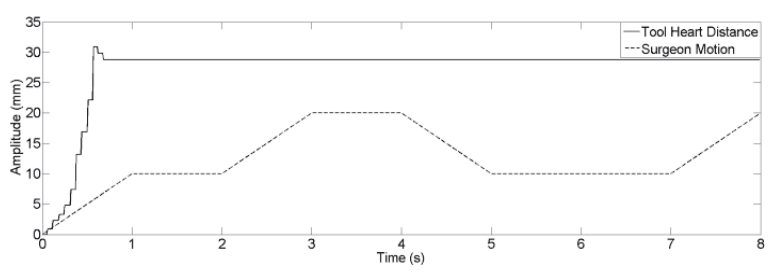

Fig. 10 The motion of the surgeon and the distance between the tool and the heart when the Smith predictor is not present.

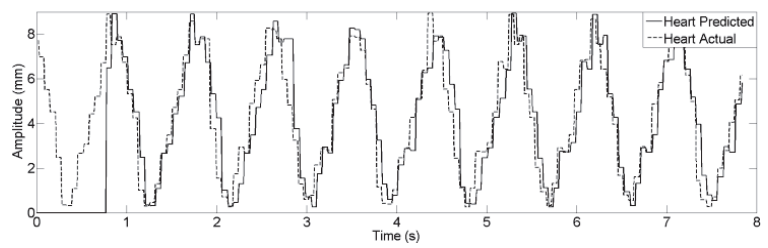

Fig. 11 A comparison of the actual and predicted heart motion when a zero-order-hold is used to increase the sampling rate of the data collected from the ultrasound images.

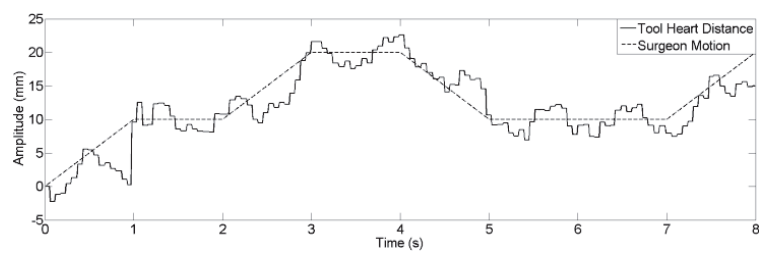

Fig. 12 The motion of the surgeon and the distance between the tool and the heart when a zero-orderhold is used to increase the sampling rate of the data collected from the ultrasound images.

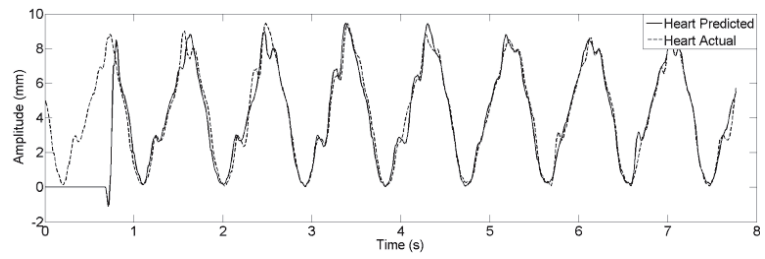

Fig. 13 A comparison of the actual and predicted heart motion when cubic interpolation is used to increase the sampling rate of the data collected from the ultrasound images.

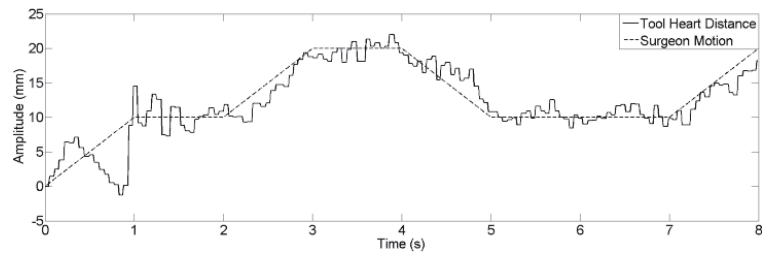

Fig. 14 The motion of the surgeon and the distance between the tool and the heart when cubic interpolation is used to increase the sampling rate of the data collected from the ultrasound images. 
the predicted heart motion does not follow the actual heart motion immediately because the motion of one heart beat must be collected as the predicted heart motion is the motion of the POI in the previous heartbeat.

\section{Concluding Remarks}

The design of a surgical system to control a surgical tool to follow the motion of the beating heart and the surgeon is challenging. First, the motion of the heart and the position of the surgical tool must be captured. Ultrasound images are chosen for this task because they can visualize both the interior surface and exterior of the heart. However, this means that there will be a time delay caused by the acquisition and processing of the ultrasound images. To account for the time delay in the control system, a Smith predictor was chosen. The heart's current motion is estimated and is treated as a disturbance. An experimental testbed is designed to test the Smith predictor. The heart motion is produced by a mechanical cam and the surgical tool is actuated by a voice coil to ensure that a fast tracking is possible. The system was tested using both a zero-order-hold and cubic interpolation to increase the sampling rate of the slowly sampled data collected from the ultrasound images. It is shown that the use of cubic interpolation reduces the tracking errors but increases the delay.

\section{References}

[1] A. Alwan: Global status report on noncommunicable diseases 2010. World Health Organization, 2011.

[2 ] M.F. Newman, J.L. Kirchner, B. Phillips-Bute, V. Gaver, H. Grocott, R.H. Jones, D.B. Mark, J.G. Reves and J.A. Blumenthal: "Longitudinal assessment of neurocognitive function after coronary-artery bypass surgery," New England Journal of Medicine, vol.344, no.6, pp.395-402, 02/08 2001.

[ 3 ] G. Reed, D. Singer, E. Picard and R. DeSanctis: "Stroke following coronary-artery bypass surgery. a case-control estimate of the risk from carotid bruits," The New England Journal of Medicine, vol.319, pp.1246-1250, 1988.

[4] R. Ginhoux, J. Gangloff, M. de Mathelin, L. Soler, M.M.A. Sanchez and J. Marescaux: "Active filtering of physiological motion in robotized surgery using predictive control," IEEE Transactions on Robotics, vol.21, no.1, p.67, 2005.

[ 5 ] P.M. Novotny, J.A. Stoll, P.E. Dupont and R.D. Howe: "Realtime visual servoing of a robot using three-dimensional ultrasound," IEEE International Conference on Robotics and Automation, pp.2655-2660, 2007.

[6] O. Bebek and M.C. Cavusoglu: "Intelligent control algorithms for robotic-assisted beating heart surgery," IEEE Transactions on Robotics, vol.23, no.3, pp.468-480, 2007.

[ 7 ] S.G. Yuen, P.M. Novotny and R.D. Howe: "Quasiperiodic predictive filtering for robot-assisted beating heart surgery," IEEE International Conference on Robotics and Automation 2008 (ICRA 2008), pp.3875-3880, 2008.

[8] F. Casamassima, C. Cavedon, P. Francescon, J. Stancanello,
M. Avanzo, S. Cora and P. Scalchi: "Use of motion tracking in stereotactic body radiotherapy: Evaluation of uncertainty in off-target dose distribution and optimization strategies," Acta Oncologica, vol.45, no.7, pp.943-947, 2006, pMID: 16982561. [Online]. Available: http://informahealthcare.com/ doi/abs/10.1080/02841860600908962

[ 9 ] D.T. Kettler, R.D. Plowes, P.M. Novotny, N.V. Vasilyev, P.J. del Nido and R.D. Howe: "An active motion compensation instrument for beating heart mitral valve surgery," IEEE/RSJ International Conference on Intelligent Robots and Systems 2007, pp.1290-1295, 2007.

[10] O.J.M. Smith: "Closer control of loops with dead time," Chemical Engineering Progress, vol.53, pp.217-219, 1957.

[11] M. Bowthorpe, M. Tavakoli, H. Becher and R. Howe: "Smith predictor-based robot control for ultrasound-guided teleoperated beating-heart surgery," IEEE Journal of Biomedical and Health Informatics, vol.18, no.1, pp.157-166, 2014.

[12] S.G. Yuen, D.T. Kettler, P.M. Novotny, R.D. Plowes and R.D. Howe: "Robotic motion compensation for beating heart intracardiac surgery," The International Journal of Robotics Research, vol.28, no.10, pp.1355-1372, 2009. [Online]. Available: http://ijr.sagepub.com/content/28/10/1355.abstract

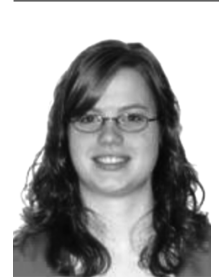

\section{Meaghan Bowthorpe}

Meaghan Bowthorpe earned her B.Sc. degree in Electrical Engineering from the University of Alberta in 2010. She is currently pursuing a $\mathrm{Ph} . \mathrm{D}$. in Electrical and Computer Engineering at the University of Alberta and is working on motion tracking algorithms for image-guided robot-assisted surgery.

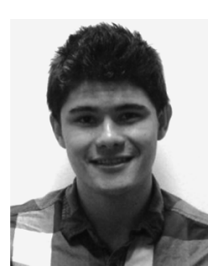

\section{Vincent Castonguay-Siu}

Vincent Castonguay-Siu is currently completing an undergraduate degree in the Department of Mechanical Engineering at the University of Alberta. This past fall he had the opportunity to work with the Telerobotics and Biorobotics Systems group led

by Dr. Tavakoli.

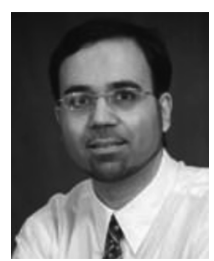

\section{Mahdi Tavakoli}

Mahdi Tavakoli received his BSc and MSc degrees in Electrical Engineering from Ferdowsi University and K.N. Toosi University, Iran, in 1996 and 1999, respectively. He then received his $\mathrm{PhD}$ degree in Electrical and Computer Engineering from the University of Western Ontario, London, ON, Canada, in 2005. In 2006, he was a post-doctoral research associate at Canadian Surgical Technologies and Advanced Robotics (CSTAR), London, ON, Canada. In 2007-2008, and prior to joining the Department of Electrical and Computer Engineering at the University of Alberta as an assistant professor, Dr. Tavakoli was an NSERC Post-Doctoral Fellow with the BioRobotics Laboratory of the School of Engineering and Applied Sciences at Harvard University, Cambridge, MA, USA. Dr. Tavakoli's research interests broadly involve the areas of robotics and systems control. Specifically, his research focuses on haptics and teleoperation control, medical robotics, and image-guided surgery. Dr. Tavakoli is the first author of the book "Haptics for Teleoperated Surgical Robotic Systems" (World Scientific, 2008). 